\title{
Diagnosis of hypothyroidism using a fuzzy rule-based expert system
}

\author{
Negar Asaad Sajadi ${ }^{\mathrm{a}}$, Shiva Borzouei ${ }^{\mathrm{b}}$, Hossein Mahjub ${ }^{\mathrm{c}}$, Maryam Farhadian $^{\mathrm{c}, *}$ \\ ${ }^{a}$ Department of Biostatistics, School of Public Health, Hamadan University of Medical Sciences, Hamadan, Iran \\ ${ }^{\mathrm{b}}$ Clinical Research Development Unit of Shahid Beheshti Hospital, Hamadan University of Medical Science, Hamadan, Iran \\ ${ }^{\mathrm{c}}$ Research Center for Health Sciences and Department of Biostatistics, School of Public Health, Hamadan University of Medical Sciences, Hamadan, Iran
}

\section{A R T I C L E I N F O}

\section{Keywords:}

Thyroid disorder

Hypothyroidism

Fuzzy rule-based expert system

Classification

Multinomial logistic regression

\begin{abstract}
A B S T R A C T
Background: Early and accurate diagnosis of many diseases is critical to their treatment. Today, the classification models based on fuzzy intelligent systems help the uncertainty conditions in medicine, as well as the classification of diseases. The main goal of this study is to diagnose the most common thyroid disorders, hypothyroidism, using a fuzzy rule-based expert system.

Methods: In this study, the data from patients who referred to Imam Khomeini Clinic and Shahid Beheshti Hospital in Hamadan west of Iran were collected. The data contain 305 subjects in three classes which are normal, subclinical hypothyroidism and hypothyroidism. Collected variables include demographic, symptoms as well as laboratory tests. In order to the diagnosis of Thyroid disorders, a fuzzy rule-based classifier was designed. Predictive performance of this model was compared with a multinomial logistic regression model in terms of the accuracy and the area under a ROC curve (AUC) as well as sensitivity and specificity.

Results: The results showed that designed fuzzy rule-based system works well in thyroid disorder prediction with about $97 \%$ accuracy. Also, the fuzzy classifier has a better performance than the logistic regression model, especially for the subclinical hypothyroidism class.

Conclusions: Fuzzy rule-based classifier by utilizing overlapping sets has improved the efficiency of classification and decision-making systems. Also, by providing the possibility of using linguistic variables in the decisionmaking process, easier interpretation, can be used by doctors who are not familiar with modeling concepts.
\end{abstract}

\section{Introduction}

The thyroid gland, a butterfly-shaped gland in front of the neck, has important roles to regulate numerous metabolic processes throughout the body. Over- or under-function of the thyroid gland known as a thyroid disorder. When an insufficient amount of thyroid hormone producing by the thyroid gland, hypothyroidism occurs. ${ }^{1,2}$

Thyroid problems are fairly common among people but are usually overlooked because their symptoms are generally mild and slow to occur. Failure to diagnose and treat any of the thyroid disorders can lead to serious complications. For example, hypothyroidism is associated with an increased risk of heart disease and hyperthyroidism causes osteoporosis. ${ }^{3}$ Diagnosis of thyroid disorder is difficult in the groups that are taking other medications or patients who are in other medical conditions. ${ }^{4}$

Considering the increasing prevalence of thyroid in the world and Iran and the irreparable harm of the disease to other organs of the body, including the eyes, heart, kidneys, etc., as well as the high economic and social costs imposed on the individual and society, it can be prevented by timely diagnosis and control of the disease as much as possible. ${ }^{5}$ Timely diagnosis and follow-up of proper treatment play an important role in preventing its complication and finally can reduce the mortality associated with the disease. ${ }^{4,5}$

Medical diagnosis in terms of classification and prediction is one of the most important applications of statistical methods. ${ }^{6}$ Classification models can be used to assist the physician to diagnose thyroid disorder more efficiently and effectively, providing scarce healthcare resources to those who need it the most.

In recent year, various machine learning methods such as neural networks, fuzzy logic, support vector machine (SVM) have been developed to solve many complex problems in the medical area. ${ }^{6-9}$ Fuzzy if-then rule-based expert systems are one of the most practical intelligent models with the high potential for managing uncertainty associated to the medical diagnosis. Zadeh (1965) introduced the theory of fuzzy sets. ${ }^{10}$

The traditional or crisp sets are based on a two-value logic: objects

\footnotetext{
* Corresponding author. P.O. Box 4171-65175, Hamadan, Iran.

E-mail addresses: n.asajadi69@yahoo.com (N. Asaad Sajadi), borzooeishiva@yahoo.com (S. Borzouei), mahjub@umsha.ac.ir (H. Mahjub), maryam_farhadian80@yahoo.com,m.farhadian@umsha.ac.ir (M. Farhadian).
} 
are either members or not members. Fuzzy logic is a multi-valued logic that is useful for solving uncertainty and vagueness in many problems, and it can address the degree of membership and degrees of truth. Fuzzy logic systems are characterized by the imprecision of the input and output variables by defining fuzzy numbers and fuzzy sets that can be expressed in linguistic variables (e.g. small, medium and large). Fuzzy inference is a process of mapping from a given input to an output using the theory of fuzzy sets. Specialist physician's knowledge is encoded as a set of explicit linguistic rules, which can be easily understood by people without technical expertise. Fuzzy systems provide the means of representing the expert knowledge of humans about the process in terms of fuzzy (IF-THEN) rules. ${ }^{10,11}$ The main goal of this study is to design of rule-based fuzzy expert system as noninvasively classifier to diagnose the most common thyroid disorders, hypothyroidism. Also, the predictive performance of fuzzy classifier compared with multinomial logistic regression model.

\section{Materials and methods}

\subsection{Setting and participants}

In this study, patients with thyroid dysfunction who referred to Imam Khomeini Clinic and Shahid Beheshti Hospital in Hamadan, in the west of Iran, for 6 months (From June to November 2017) were examined. This study was approved by the Ethics Committee of Hamadan University of Medical Sciences with IR.UMSHA.REC.1396. 242 codes All subjects were interviewed by a trained research student or specialist physician and completed a structured questionnaire designed in consultation with two endocrinologists. All subjects gave informed written consent to participate. The study was approved by a research ethics committee.

The data contain 305 subjects in three classes which are normal, subclinical hypothyroidism and hypothyroidism. Fifty patients belong to sub-Hypo class, 155 patients belong to Hypo class and 105 of them belong to a Normal class.

Collected variables include demographic variables, variables associated with symptoms of hypothyroidism (including: fatigue, sleepiness, constipation, feeling cold, fluid retention, weight gain, reduce appetite and menstrual irregularities in women) as well as variables related to laboratory tests (including: T4 (mIU/L), TSH (mIU/L)).

The score. Hypo variable are defined as follows if the person has the desired symptom, the value is one, and if the symptom not exists, the value takes zero. The score. Hypo variable is defined as the sum of the symptoms for each individual. So when the value of the score. Hypo is three for an individual, it means that the person has three signs.

\subsection{Multinomial logistic regression}

Logistic regression is a statistical model that fits the log odds of the dependent variable to a linear combination of the independent variables. It is used mainly for binary responses, although there are extensions for multi-class responses as well. In logistic regression the probability of occurrence (success $=1$ ) or non-occurrence of an event (failure $=0$ ) for a given person is expressed by using the following equation:

$P\left(y^{*}=1 \mid x^{*}, \beta\right)=\left(1+\exp \left(-x^{*} \beta\right)\right)^{-1}$

Multinomial Logistic Regression used when the dependent variable is nominal with more than two levels. In multinomial logistic regression, given the multinomial class variable $\left(y_{i}\right)$ with $\mathrm{j}$ categories and $\mathrm{p}$ dimensional predictor variables $\left(x_{p}\right)$, forecast whether a future data point $y^{*}$ observed at the predictor $\left(x^{*}\right)$ will be belong to which class variable. The probability of belonging to the category $j$ for a given person is expressed by using the following equation:

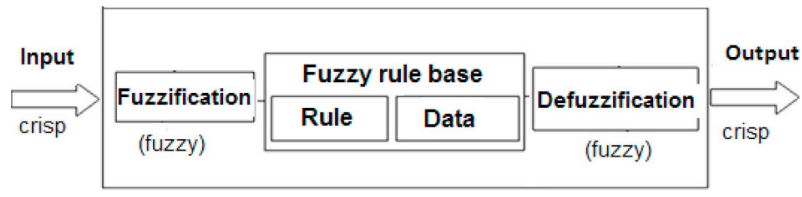

Fig. 1. Block diagram for a fuzzy rule based System.

$P\left(y^{*}=j \mid x^{*}, \beta\right)=e^{x^{*} \beta_{j}} / \sum_{k=1}^{J} e^{x^{*} \beta_{k}}$

The unknown parameters of multinomial logistic regression are typically jointly estimated by maximum a posteriori (MAP) estimation, which is an extension of maximum likelihood. ${ }^{12}$

\subsection{Design of Mamdani-type fuzzy rule based expert systems}

A fuzzy inference system with crisp inputs and outputs implements a nonlinear mapping from its inputs space to output space. This mapping is done by a number of fuzzy if-then rules. The antecedent of a rule defines a fuzzy region in the input space, while the consequent specifies the output in the fuzzy region. ${ }^{13}$ Basically, a fuzzy inference system is composed of five functional blocks as shown in Fig. 1. The structure of the fuzzy inference system is described as follows.

- A database including: identify the input, output variables and defining the fuzzy set for each variable, select the membership functions and parameters of these for graphically presenting inputs and output variables. It is noted that uncertainty in a fuzzy set can be solved by membership functions. The membership value of a member such as $\mathrm{x}$ in the functions between 0 and 1 . This means that a function get an entry such as the $\mathrm{x}$ and it's the interval $[0,1]$ defines.

- A rule base containing a number of fuzzy if-then rules corresponding to these membership functions. A fuzzy rule set establishes a relationship between different input fuzzy sets and output sets.

- Fuzzification: convert the input crisp values into linguistic variables like low, middle and high. Fuzzification of the input variables is made by selecting membership functions which determined by help both of the expert-doctor and literature functions.

- Defuzzification converting linguistic variables into crisp value using the center of gravity (COG) method to display the final output. In this stage, truth degrees of the rules are determined for each rule by the aid of the min and then by taking max between working rules. The crisp value of the output variable is calculated by the method center of gravity defuzzifier.

It is noted that, in this study or the inference mechanism, the Mamdani max-min inference was used. Developed fuzzy inference system (FIS) has a structure shown as in Fig. 2. The membership function associated with the inputs and output variables presented in Fig. 3. The rules of the fuzzy expert system for thyroid disorders diagnosis are given in Fig. 4.

\subsection{Evaluation of the proposed fuzzy system}

To investigate the quality of prediction accuracy, sensitivity, specificity and the area under the ROC curve (AUC) are used. AUC is a measure of model discrimination (i.e., how well the model separates subjects who did and did not experience an event).

\subsubsection{Train and test sets}

The training set is used to obtain the regression weights. The performance of the model should be tested on the test set. To obtain a more realistic estimate of how the model would perform with unseen data, a side part of the original data not include in the training process must be 
AIS Editor: HypoFIS
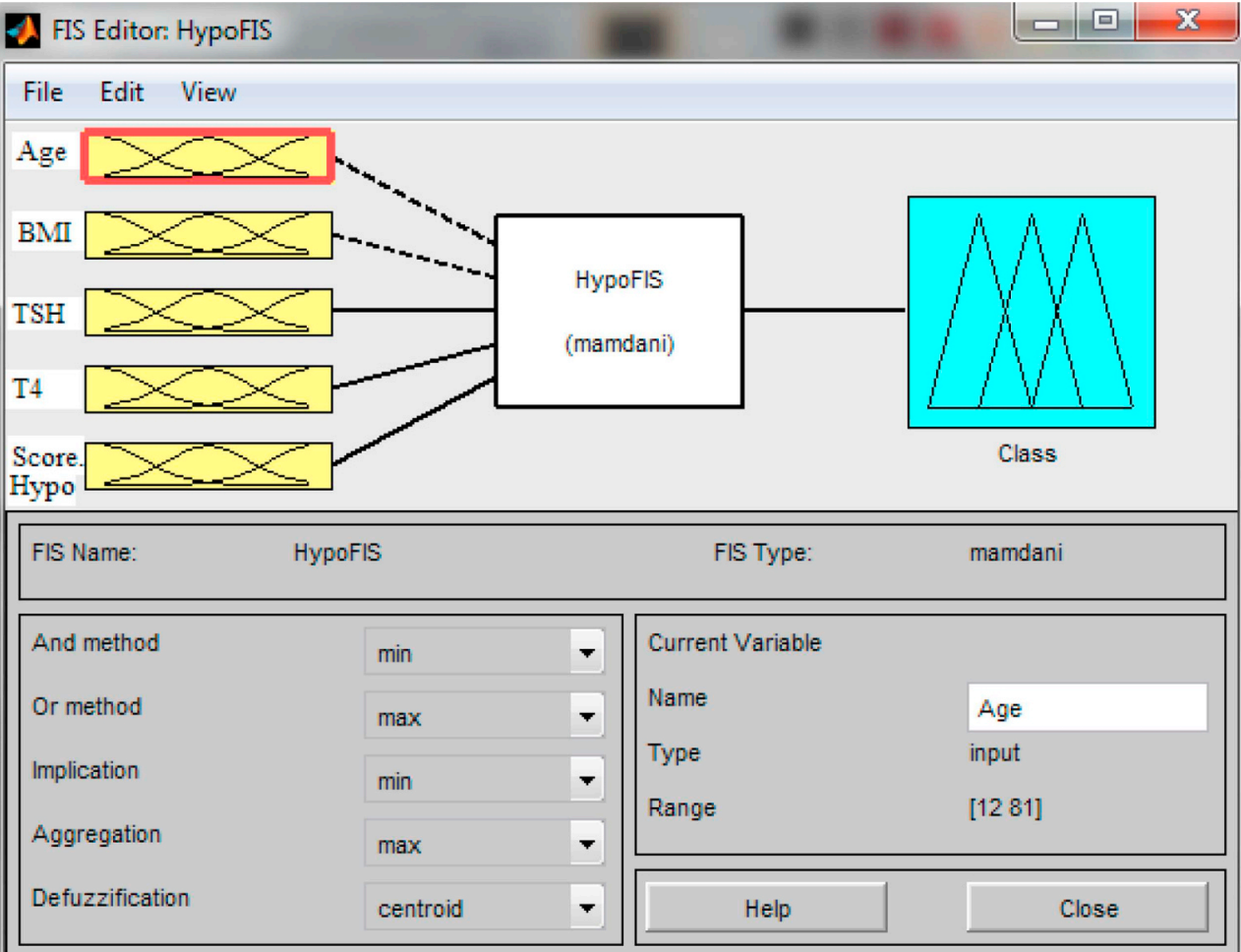

System "HypoFIS": 5 inputs, 1 output, and 8 rules

Fig. 2. Developed FIS structure for hypothyroid disorder diagnosis.

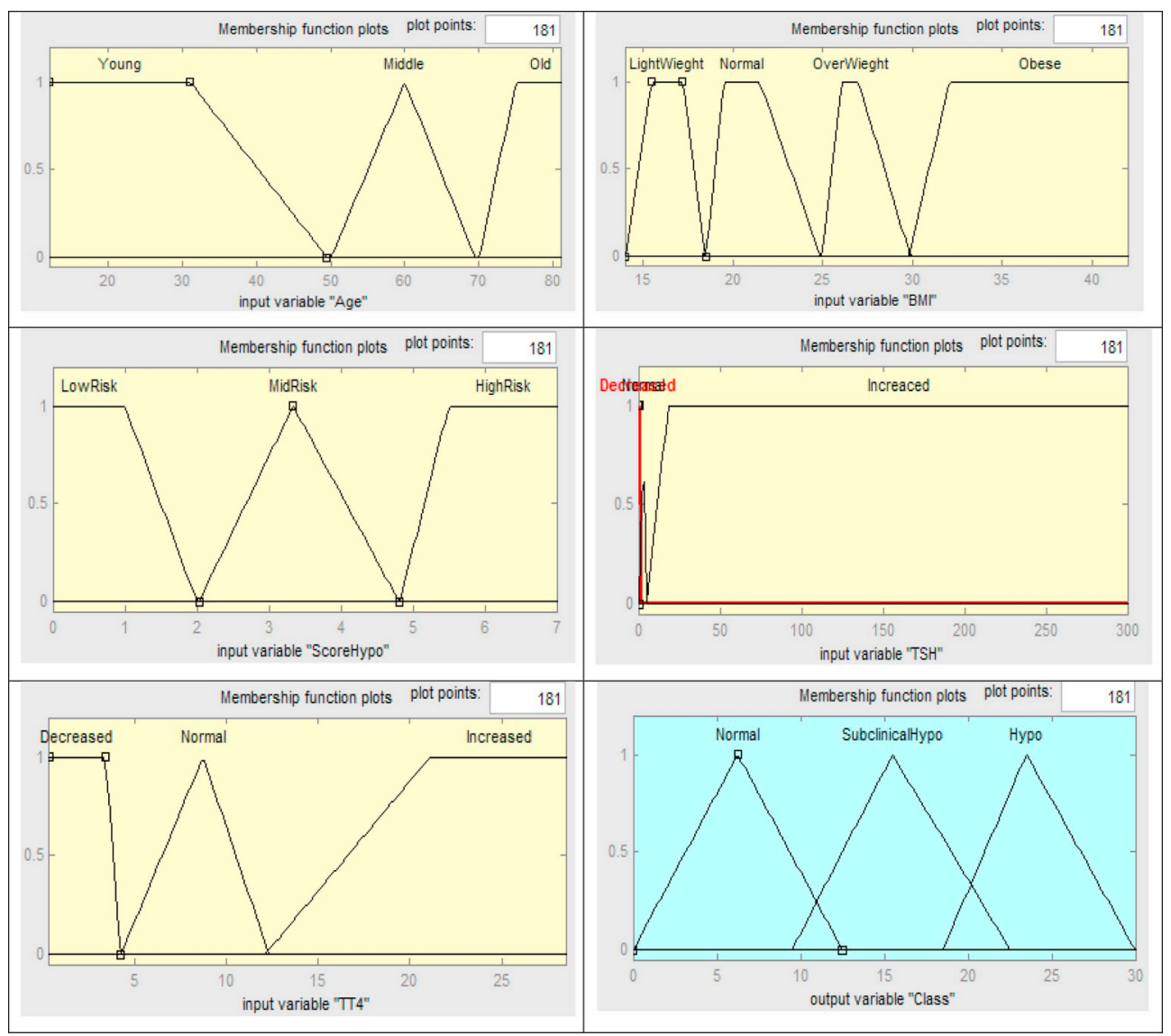

Fig. 3. Membership functions for input (Age, BMI, T4, TSH, Score. Hypo) and output (Thyroid disorder status; Class) variables. 


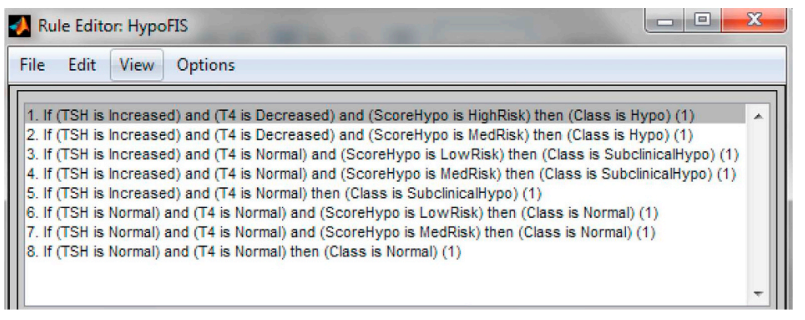

Fig. 4. Rule set of the expert fuzzy system for thyroid disorders diagnosis.

set. This dataset is known as the test set. ${ }^{14}$ The testing sets have the actual category and this allows to compare the predicted category with the actual category and thus assess the predictive performance of a model. All analysis implemented in the fuzzy toolbox of MATLAB software.

It is noted that, in order to evaluate the predictive ability of proposed fuzzy rule base classifier and multinomial logistic regression model the data set was randomly divided into training $(7 / 10$ of the data) and test (3/10 of the data) sets. It is noted that in the current study to obtain comparable results the same training and testing parts used for a different fuzzy and logistic models.

\section{Results}

The frequency distribution of gender and family history, as well as the comparison of mean age (year), body mass index, TSH, T4 and score. Hypo are presented in Table 1 . The results show that in the group of patients with subclinical hypothyroidism and hypothyroidism $(80 \%$ and $91 \%$, respectively) have been women, also $78 \%$ of the patients were female in the healthy group. Among people with subclinical hypothyroidism, $60 \%$ had a family history of thyroid disease, and $64 \%$ of those with hyperthyroidism had a family history of thyroid disease. However, in people who have been diagnosed with normal, there is a family history of thyroid disease in $21 \%$ of people. Comparison of mean age, body mass index, TSH, T4 and score. Hypo variables in three groups indicate that there is a statistically significant difference in relation to the mean of all variables in the three groups except BMI (kg/ $\mathrm{m}^{2}$ ). The evaluation of the score. Hypo was $3.69 \pm 1.68$ in the hypothyroidism group and $2.34 \pm 1.7$ in the subclinical hypothyroidism group, and in the normal group, it was $0.06 \pm 0.23$.

In the proposed fuzzy rule-based classifier variables including age, BMI, T4, TSH and score. Hypo are used as input and thyroid status or class is used as output. The fuzzy output variable (Class) has been represented using 3 fuzzy sets. The range of the membership function is from 0 to 30 . The class includes hyperthyroid (range of $0-12.5$ with
Table 2

Predictive performance of fuzzy rule-based system and multinomial logistic regression based on the test and train sets.

\begin{tabular}{|c|c|c|c|c|c|c|c|}
\hline Classifier & & ACC & Mean AUC & & Normal & Sub-Hypo & Нypo \\
\hline \multirow[t]{3}{*}{ Fuzzy } & Test & 0.967 & 0.971 & Sensitivity & 1.000 & 0.825 & 1.000 \\
\hline & & & & Specificity & 0.964 & 1.000 & 0.983 \\
\hline & & & & Accuracy & 0.982 & 0.912 & 0.991 \\
\hline \multirow[t]{3}{*}{ Logistic } & Test & 0.817 & 0.843 & Sensitivity & 0.987 & 0.210 & 0.906 \\
\hline & & & & Specificity & 0.937 & 0.967 & 0.779 \\
\hline & & & & Accuracy & 0.962 & 0.589 & 0.843 \\
\hline \multirow[t]{3}{*}{ Fuzzy } & Train & 0.987 & 0.983 & Sensitivity & 1.000 & 0.894 & 1.000 \\
\hline & & & & Specificity & 0.985 & 1.000 & 0.977 \\
\hline & & & & Accuracy & 0.992 & 0.947 & 0.989 \\
\hline \multirow[t]{3}{*}{ Logistic } & Train & 0.790 & 0.820 & Sensitivity & 0.857 & 0.286 & 0.826 \\
\hline & & & & Specificity & 0.981 & 0.865 & 0.829 \\
\hline & & & & Accuracy & 0.919 & 0.575 & 0.827 \\
\hline
\end{tabular}

triangular shape) Normal (range of 7.5-22.5) and hypothyroid (range of 17.5-30). Linguistic variables along with membership functions for all input and output variables presented in Fig. 3. For example, linguistic variables for age including Young, Middle Age, and Old. The proposed rule base fuzzy expert system consists of 12 fuzzy rules, which are extracted using the knowledge of two medical experts.

The prediction accuracy along with, overall accuracy, sensitivity, and specificity on the test and train sets were provided in Table 2. The results showed that proposed FIS worked well in hypothyroid disorder prediction.

\section{Discussion}

In this study, a fuzzy rule-based expert system for diagnosing hypothyroidism disorder was developed. According to the findings of this study, interpretable fuzzy rule-based classification has a high classification accuracy rate. Also, the fuzzy classifier has a better performance than the logistic regression model, especially for the subclinical hypothyroidism class. This system can assist experts' decision making for diagnosis of thyroid disorder. Because of fuzzy system high interpretability, they are close to human's language, easy to interact with human experts.

Using a precise model for predicting thyroid disorder can be helpful for young doctors to use it as a supplementary model for predicting thyroid. Also, in many less developed countries due to lack of expertise and equipment, the number of physicians per ordinary person is very low. In such a situation, the use of an accurate predictive model can be very beneficial.

Also, medical decision-making based on expert systems such as

Table 1

Comparison of demographic, laboratory and symptomatic variables in three groups.

\begin{tabular}{|c|c|c|c|c|c|c|c|c|}
\hline & & & & Group & & & & \\
\hline \multirow[t]{2}{*}{ Variable } & & \multicolumn{2}{|l|}{ Healthy } & \multicolumn{2}{|l|}{ Sub-clinical Hypo } & \multicolumn{2}{|l|}{ Hypothyroidism } & \multirow[t]{2}{*}{ P-value ${ }^{a}$} \\
\hline & & Mean \pm SD & & Mean \pm SD & & Mean $\pm S D$ & & \\
\hline Age & & $47.08 \pm 17.21$ & & $37.32 \pm 13.63$ & & $41.27 \pm 12.85$ & & $<0.001$ \\
\hline BMI & & $26.02 \pm 4.08$ & & $29.93 \pm 3.74$ & & $27.07 \pm 4.67$ & & 0.74 \\
\hline TSH & & $2.55 \pm 1.43$ & & $8.32 \pm 5.49$ & & $19.55 \pm 23.73$ & & $<0.001$ \\
\hline $\mathrm{T} 4$ & & $9.86 \pm 2.77$ & & $7.64 \pm 3.74$ & & $5.84 \pm 2.83$ & & $<0.001$ \\
\hline \multirow[t]{2}{*}{ Score.Hypo } & & $0.06 \pm 0.23$ & & $2.34 \pm 1.70$ & & $3.69 \pm 1.68$ & & $<0.001$ \\
\hline & & $\mathrm{N}$ & $\%$ & $\mathrm{~N}$ & $\%$ & $\mathrm{~N}$ & $\%$ & Total \\
\hline \multirow[t]{2}{*}{ Sex } & Female & 82 & 31.7 & 40 & 15.4 & 137 & 52.9 & 259 \\
\hline & Male & 23 & 50.0 & 10 & 21.7 & 13 & 28.3 & 46 \\
\hline \multirow[t]{2}{*}{ History } & Yes & 83 & 39.7 & 30 & 14.3 & 96 & 46.0 & 209 \\
\hline & No & 22 & 22.9 & 20 & 20.8 & 54 & 56.3 & 96 \\
\hline
\end{tabular}

\footnotetext{
a ANOVA test.
} 
fuzzy rule-based models has many benefits, for example, data collection can be done through the patient itself. In addition to numerical data, medical images and X-rays can also be imported into such systems. These decisions are more accurate than doctors' decision making, which is affected by fatigue and human error, and they are also more likely to get precise results. ${ }^{13}$

In the literature, thyroid disease data set from the University of California Irvine (UCI) Machine Learning Repository's website is widely used in thyroid studies for the classification propose. ${ }^{15}$ In most of these studies, it has been attempted to increase the accuracy of diagnosis by applying different classification methods. ${ }^{16-25}$ In the present study, a real dataset was used to predict thyroid disorder. One of the main drawbacks of most of the presented approaches is that they have not been evaluated on real datasets. Consequently, their performance in real circumstances is not clear. Many studies have only used laboratory variables for the diagnosis of thyroid disorder. ${ }^{16-25}$ As far as we know, in limited studies, both sets of laboratory tests and some of the symptoms variables were used in the classification model. ${ }^{30}$

Various research works in the field of thyroid classification based on different data mining techniques are mentioned. ${ }^{19}$ For example, Azar in 2012 presented an expert system based on neural-fuzzy rules for diagnosis of thyroid disease. A thyroid data from this study was extracted from the UCI site. The number of subjects was 215 including 150 hyperthyroid, 35 hypothyroidisms and 30 healthy. The results indicated that the classification accuracy without feature selection was $98.6 \%$ and $97.67 \%$ for training and testing data sets, respectively. ${ }^{26}$

Gharehchopogh in 2013, proposed a Multi-Layer Perceptron (MLP) ANN using back propagation learning for the automatic diagnosis of thyroid disease. The number of subjects was 215 . For each person, 5 laboratory test were extracted and the outcome of the person's status has three levels. These states are Normal, Hyperthyroidism and Hypothyroidism. In this study, a MLP model with a hidden layer and Logsig activation function for hidden layer and 6 neurons in the hidden layer, was proposed. The accuracy of the proposed models based on the test data set was reported as $98.6 \% .^{27}$

Turanoglu-Bekar in 2016, compared different decision Tree algorithms for diagnosing thyroid disease according to six performance metrics. The input variables including TSH, T4, T3. The output variable includes five class. The results of study showed that NBTree algorithm with $75 \%$ accuracy, has a highest accuracy rate. ${ }^{28}$

Khanale in 2011 presented a fuzzy Mamdani inference system, including 18 if-then rules to provide a diagnosis of hypothyroidism. In this system, 45 people were examined and the input variables included symptom score expressed as a percentage of the severity of symptoms, (2) T4 and (3) TSH. The system output is the actual diagnosis of the patient which gives the severity of the hypothyroidism which is divided into four types. The triangular and trapezoidal membership function has been used. The outcome is a healthy or type of thyroid abnormality that has three levels (subclinical malnutrition, Secondary). The accuracy of the system's prediction is about $88 \%$.

Kelus in 2008, presented an expert system for diagnosis of thyroid disease, called the ESTDD. In this study, fuzzy rules extracted using neural networks method. Thyroid data from the UCI site that contains 250 individuals was used. The system has 5 input variables (including laboratory variables associated with thyroid hormones) and one output variable including the status of the individual (healthy, thyroid deficiency and hyperthyroidism). The designed system had a precision of $95 \% .^{29}$

Biyouki in 2015 designed a fuzzy rule-based expert system including three rules for diagnosis of thyroid disease. In this study, two data sets related to the UCI database contains 215 samples, 3 classes (150 instances of Euthyroid class, 35 instances of hyperthyroidism class and 30 instances of hypothyroidism class.) and for each sample five features (T3-resin uptake test, T4, T3, TSH and Maximal absolute difference of TSH) were used. The classification accuracy of the proposed system for diagnosis of thyroid disease was obtained about $89.09 \% .{ }^{30}$
These comparisons indicate that the results of the proposed model are in many cases similar to other studies and in some cases even improved. Therefore, while the results of the study are satisfactory, further review is needed to evaluate the performance of other methods of extracting the fuzzy rules. However, one of the limitations of this study is that to extract the rules, all the input variables have the same weight. Therefore, the input weighting method to extract the rules was recommended in the future study.

With the advent of expert systems, the effects of this innovation in the field of medicine are increasing day by day. Medical decision making can sometimes be difficult. Classification systems that are used in medical decision-making will examine medical data in the shortest time and with more precise details.

\section{Conclusion}

The results of this study indicate that designed fuzzy rule-based systems model based on clinical symptom and laboratory variables have an acceptable predictive performance for diagnosis hypothyroidism disorder. This system can be used by the young specialist doctors for helping in diagnosis and by the students with the goal of learning and self-evaluation.

\section{Conflicts of interest}

The authors have no conflicts of interest to declare for this study.

\section{Acknowledgements}

This study was supported by the Hamadan University of Medical Sciences. The authors are indebted to the patients that participated in this project.

\section{Appendix A. Supplementary data}

Supplementary data to this article can be found online at https:// doi.org/10.1016/j.cegh.2018.11.007.

\section{References}

1. Dayan CM. Interpretation of thyroid function tests. Lancet. 2001;357(9256):619-624 2. Kalra S, Khandelwal SK, Goyal A. Clinical scoring scales in thyroidology: a com pendium. Indian J Endocr Metab. 2011;15:S89-S94.

3. Monaco F. Classification of thyroid diseases: suggestions for a revision. $J$ Clin Endocrinol Metab. 2003;88(4):1428-1432.

4. Mbah AU, Ejim EC, Onodugo OD, et al. Two logistic models for the prediction of hypothyroidism in pregnancy. BMC Res Notes. 2011;4:205.

5. Khiew KF, Wang TI, Lin MYS, et al. Prediction of hypothyroidism disease by data mining technique. J Data Sci. 2016;14:97-116.

6. Farhadian M, Mahjub H, Poorolajal J, et al. Predicting 5-year survival status of patients with breast cancer based on supervised wavelet method. Osong Public Health Res Perspect. 2014;5(6):324-332. https://doi.org/10.1016/j.phrp.2014.09.002.

7. Kazemi M, Moghimbeigi A, Kiani J, et al. Diabetic peripheral neuropathy class prediction by multi category support vector machine model: a cross-sectional study. Epidemiol Health. 2016;38:e2016011.

8. Aliabadi M, Farhadian M, Darvishi E. Prediction of hearing loss among the noiseexposed workers in a steel factory using artificial intelligence approach. Int Arch Occup Environ Health. 2015;88(6).

9. Shankar K, Lakshmanaprabu SK, Gupta D, et al. Optimal feature-based multi-kernel SVM approach for thyroid disease classification. J Supercomput. 2018. https://doi. org/10.1007/s11227-018-2469-4.

10. Yen J, Langari R. Fuzzy Logic: Intelligence, Control, and Information. Upper Saddle River, NJ: Prentice Hall; 1999 Dec.

11. Zadeh LA. The concept of a linguistic variable and its application to approximate reasoning-I. Inf Sci. 1975 Jan 1;8(3):199-249.

12. Agresti A. Categorical Data Analysis. second ed. New York: John Wiley \& Sons; 2003

13. Phuong NH, Kreinovich V. Fuzzy logic and its applications in medicine. Int J Med Inf. 2001;62(2-3):165-173

14. Hastie T, Tibshirani R, Friedman J. The Elements of Statistical Learning: Data Mining Inference, and Prediction. second ed. New York: Springer; 2009.

15. Asuncion A, Newman D. UCI Machine Learning Repository. 2007; 2007.

16. Ozyılmaz L, Yıldırım T. Diagnosis of thyroid disease using artificial neural network methods. Proceedings of ICONIP'02 9th International Conference on Neural Information 
Processing. Singapore: Orchid Country Club; 2002:2033-2036. https://doi.org/10. 1109/ICONIP.2002.1199031.

17. Polat K, Şahan S, Güneş S. A novel hybrid method based on artificial immune recognition system (AIRS) with fuzzy weighted pre-processing for thyroid disease diagnosis. Expert Syst Appl. 2007 May 1;32(4):1141-1147.

18. Keles A, Keles A. ESTDD: expert system for thyroid diseases diagnosis. Expert Syst Appl. 2008;34(1):242-246.

19. Temurtas F. A comparative study on thyroid disease diagnosis using neural networks. Expert Syst Appl. 2009 Jan 1;36(1):944-949.

20. Dogantekin E, Dogantekin A, Avci D. An automatic diagnosis system based on thyroid gland: ADSTG. Expert Syst Appl. 2010 Sep 1;37(9):6368-6372.

21. Chen HL, Yang B, Wang G, et al. A three-stage expert system based on support vector machines for thyroid disease diagnosis. J Med Syst. 2012;36:1953-1963.

22. Kousarrizi MRN, Seiti F, Teshnehlab M. An experimental comparative study on thyroid disease diagnosis based on feature subset selection and classification. Int $J$ Electr Comput Sci IJECS-IJENS. 2012 Feb;12(01):13-19.

23. Radwan E, Assiri AMA. Thyroid diagnosis based technique on rough sets with modified similarity relation. Thyroid. 2013 Oct;4(10).

24. Pandey S, Tiwari A, Shrivas AK, et al. Thyroid classification using ensemble model with feature selection. Int J Comput Sci Inf Technol. 2015;6(3):2395-2398.

25. Razia S, Rao MRN. Machine learning techniques for thyroid disease diagnosis-a review. Indian J Sci Technol. 2016;9(28):1-9.

26. Azar AT, Hassanien AE, Kim TH. Expert system based on neural-fuzzy rules for thyroid diseases diagnosis. In: International Conference on Bio-science and Biotechnology (BSBT 2012), 16-19 Dec 2012, Korea, Vol 353 of the Communications in Computer and Information Science Series. Springer, 94-105. ISBN: 978-3-64235520-2. DOI: 10.1007/978-3-642-35521-9_13.

27. Gharehchopogh FS, Molany M, Mokri FD. Using artificial neural network in diagnosis of thyroid disease: a case study. Int J Comput Sci Appl (IJCSA). 2013;3:49-61.

28. Turanoglu-Bekar E, Ulutagay G, Kantarcı-Savas S. Classification of thyroid disease by using data mining models: a comparison of decision tree algorithms. Oxford J Intell Decis Data Sci. 2016;2016(2):13-28. https://doi.org/10.5899/2016/ojids-00002.

29. Khanale PB, Ambilwade RP. A fuzzy inference system for diagnosis of hypothyroidism. J Artif Intell. 2011;4(1):45-54.

30. Biyouki SA, Turksen IB, Fazel Zarandi MH. Fuzzy rule-based expert system for diagnosis of thyroid disease. IEEE Conference on Computational Intelligence in Bioinformatics and Computational Biology (CIBCB). 12-15 Aug. 2015. Niagara Falls, ON, Canada. IEEE; 2015https://doi.org/10.1109/CIBCB.2015.7300333. 